
and Mehar Ali Malik

\title{
Szeged-type indices of subdivision vertex-edge join (SVE-join)
}

https://doi.org/10.1515/mgmc-2021-0011

received November 12, 2020; accepted March 09, 2021

Abstract: In this article, we compute the vertex Padmakar-Ivan $\left(P I_{v}\right)$ index, vertex Szeged $\left(S z_{v}\right)$ index, edge Padmakar-Ivan $\left(P I_{e}\right)$ index, edge Szeged $(S z)$ index, weighted vertex Padmakar-Ivan $\left(w P I_{v}\right)$ index, and weighted vertex Szeged $\left(w S z_{v}\right)$ index of a graph product called subdivision vertex-edge join of graphs.

Keywords: topological indices, distance in graphs, graph products

\section{Introduction, basic definitions, and notations}

A molecule is represented by a graphical method which shows the relationship between the atoms and bonds in that molecule. Such a representation is called a molecular graph. We denote a molecular graph by $\Gamma$. The set containing all the atoms is called the vertex set of the molecular graph $\Gamma$, and denoted by $V(\Gamma)$. Similarly, the set containing all the edges of $\Gamma$ is denoted by $E(\Gamma)$. The quantity $|V(\Gamma)|$ represents the number of atoms in $\Gamma$, and it is called the order of $\Gamma$. Similarly, the cardinality

\footnotetext{
* Corresponding author: Yu-Ming Chu, Department of Mathematics, Huzhou University, Huzhou 313000, China; Hunan Provincial Key Laboratory of Mathematical Modeling and Analysis in Engineering, Chengsha University of Science and Tehnology, Changsha 410114, China; e-mail: chuyuming2005@126.com

Syed Sheraz Asghar and Muhammad Ahsan Binyamin: Department of Mathematics, Government College University, Faisalabad, Pakistan

Shehnaz Akhtar: Department of Mathematics, School of Natural Sciences, National University of Sciences and Technology, H-12, Islamabad, Pakistan

Mehar Ali Malik: Department of Mathematics, Riphah Institute of Computing and Applied Sciences Riphah International University, Lahore, Pakistan
}

$|E(\Gamma)|$ represents the size of $\Gamma$, which is the number of chemical bonds or edges in $\Gamma$. Let $|V(\Gamma)|=n$ and $|E(\Gamma)|=$ $m$. The distance $d_{\Gamma}(v, w)$ between two vertices $v, w \in V(\Gamma)$ is the length of a path of minimal length joining $v$ with $w$ in $\Gamma$. Let $u, x, y \in V(\Gamma)$ and $e=x y \in E(T)$. The distance between $u$ and $e$ is defined as $d_{\Gamma}(u, e)=\min \left\{d_{\Gamma}(u, x), d_{\Gamma}\right.$ $(u, y)\}$. A set $S \subseteq E(\Gamma)$ is called an edge-cut of a connected graph $\Gamma$ if $\Gamma-S$ is disconnected. For $e=u v \in E(\Gamma)$, the cardinalities of the sets containing vertices or edges in $\Gamma$, which are closer to one end-vertex of $e$ are important to study. These number are obtained from the sets defined as follows:

$$
\begin{aligned}
& N_{u}(e \mid \Gamma)=\left\{x \in V(\Gamma) \mid d_{\Gamma}(u, x)<d_{\Gamma}(v, x)\right\} \\
& M_{u}(e \mid \Gamma)=\left\{f \in E(\Gamma) \mid d_{\Gamma}(u, f)<d_{\Gamma}(v, f)\right\}
\end{aligned}
$$

The cardinalities $\left|N_{u}(e \mid \Gamma)\right|$ and $\left|M_{u}(e \mid \Gamma)\right|$ are denoted by $n_{u}(e \mid \Gamma)$ and $m_{u}(e \mid \Gamma)$, respectively. The quantities $n_{v}$ $(e \mid \Gamma)$ and $m_{v}(e \mid \Gamma)$ are defined analogously. Similarly, we define the sets $N_{0}(e \mid \Gamma)$ and $M_{0}(e \mid \Gamma)$ of vertices and edges that are equidistant from both ends of the edge $e=u v$ as follows:

$$
\begin{aligned}
& N_{0}(e \mid \Gamma)=\left\{x \in V(\Gamma) \mid d_{\Gamma}(u, x)=d_{\Gamma}(v, x)\right\} \\
& M_{0}(e \mid \Gamma)=\left\{f \in E(\Gamma) \mid d_{\Gamma}(u, f)=d_{\Gamma}(v, f)\right\}
\end{aligned}
$$

Clearly $\left\{N_{u}, N_{v}, N_{0}\right\}$ define the vertex partition of $\Gamma$ with respect to an edge $e \in E(\Gamma)$. Thus $n_{u}+n_{v}+n_{0}=n$. Observe that in any bipartite graph, $n_{0}=0$.

\section{Topological indices}

A molecular or topological descriptor/index is a quantity that correlates some properties of a molecule by a constant. These properties include physical or chemical properties or biological reactivity in molecules. The definitions and analysis of these topological indices uses several tools from graph theory and heavily depends on it. A standard 
procedure in QSAR/QSPR studies is to find applications of molecular descriptors. The relations between the physicochemical properties of a chemical compounds and the molecular connectivity of the same compound are used in QSAR/QSPR studies by using graph techniques. The same process is adopted in computer-aided drug discovery and predictive toxicology. As a result, many molecular topological descriptors emerge from the successful relationships which are found (Azari and Iranmanesh, 2013; Bajaj et al., 2006; Balasubramanian, 1985a, 1985b; Basak et al., 2000).

Wiener (1947) defined the first topological index called the Wiener index as a structural descriptor for some alkanes. This index is defined as the sum of all pairwise distances between vertices of a graph. This index has been intensively studied in theoretical chemistry. It has found numerous applications in pharmacology, and many physico-chemical properties of organic molecules, see books (Balaban et al., 1983; Diudea, 2001) and survey (Dobrynin and Entringer, 2011). For a connected graph $\Gamma$, the Wiener index $W(\Gamma)$ and its equivalent form for a tree $T$ is defined as follows.

$$
W(\Gamma)=\sum_{\{u, v\} \subseteq V(\Gamma)} d_{\Gamma}(u, v), \quad W(T)=\sum_{u v \in E(T)} n_{u} n_{v}
$$

Platt (1952) used the term Wiener number for it and the same has been exclusively used ever since. Hundreds of molecular descriptors have been defined during the past decade. All of these indices are candidates for possible applications in molecular structure-property studies and many are actually used in QSAR/QSPR studies (Devillers and Balaban, 1999; Gupta et al., 2002; Hansch and Leo, 1996). The advancement in the quantitative study of structure-property relationships requires investigation of new indices that are capable of correlating different properties of chemical compounds. These descriptors are tested with linear or multivariate regressions for their applications in pharmacology and engineering sciences (Randić, 2004).

In this paper we study several Szeged-type topological indices of SVE-join of graphs. First we introduce the basic definitions of these indices and notations in the next section.

\section{Szeged-type indices}

In this section, we introduce some Szeged-type topological indices that are defined as the number of vertices or edges that lye on either side of a vertex or an edge. These indices are distance based indices and are extensively studied in the literature for their applications (Gutman et al., 1995). Now we define some Szeged indices as follows.

Gutman (1994) extended the idea of the Wiener index to any connected graph and named it as Szeged index $\left(S z_{v}\right)$ defined in the following way.

$$
S z_{v}(\Gamma)=\sum_{e=x y \in E(\Gamma)} \mathrm{n}_{x}(e \mid \Gamma) \mathrm{n}_{y}(e \mid \Gamma) .
$$

The vertex Padmakar-Ivan $\left(P I_{v}\right)$ index of a graph $\Gamma$ was defined by Khalifeh et al. (2009), as follows:

$$
P I_{v}(\Gamma)=\sum_{e=x y \in E(\Gamma)}\left(\mathrm{n}_{x}(e \mid \Gamma)+\mathrm{n}_{y}(e \mid \Gamma)\right) .
$$

Khadikar et al. (2001) defined the Padmakar-Ivan or $P I_{e}$ index as follows:

$$
P I_{e}(\Gamma)=\sum_{e=x y \in E(\Gamma)}\left(\mathrm{m}_{x}(e \mid \Gamma)+\mathrm{m}_{y}(e \mid \Gamma)\right) .
$$

Gutman and Ashrafi (2008) defined the index $\left(S z_{e}\right)$ as the edge type of the already defined Szeged index as:

$$
S z_{e}(\Gamma)=\sum_{e=x y \in E(\Gamma)} \mathrm{m}_{x}(e \mid \Gamma) \mathrm{m}_{y}(e \mid \Gamma) .
$$

The weighted vertex Padmakar-Ivan $\left(w P I_{v}\right)$ index of a graph $\Gamma$ was defined by Ilic and Milosavljevic (2013) as described below:

$$
P I_{v}(\Gamma)=\sum_{e=x y \in E(\Gamma)}\left(d_{\Gamma}(x)+d_{\Gamma}(y)\right)\left(\mathrm{n}_{x}(e \mid \Gamma)+\mathrm{n}_{y}(e \mid \Gamma)\right) .
$$

The weighted vertex Szeged index $\left(w S z_{v}\right)$ defined in Ilic and Milosavljevic (2013) is given by:

$$
S z_{v}(\Gamma)=\sum_{e=x y \in E(\Gamma)}\left(d_{\Gamma}(x)+d_{\Gamma}(y)\right) \mathrm{n}_{x}(e \mid \Gamma) \mathrm{n}_{y}(e \mid \Gamma) .
$$

\section{SVE-join of graphs}

Graph operations are used to construct new graphs whose structure is defined in terms of the structural properties of underlying graphs. In this section, we define a recently defined graph operation known as subdivision vertex edge join of graphs. It is denoted by SVE-join of graphs. This operation is applied on three graphs and results in one product graphs. The study of topological indices for several graph operations has been carried out in the literature, for examples see: Ashrafi et al. (2010), Das et al. (2013), De et al. (2016), Imran et al. (2020), 

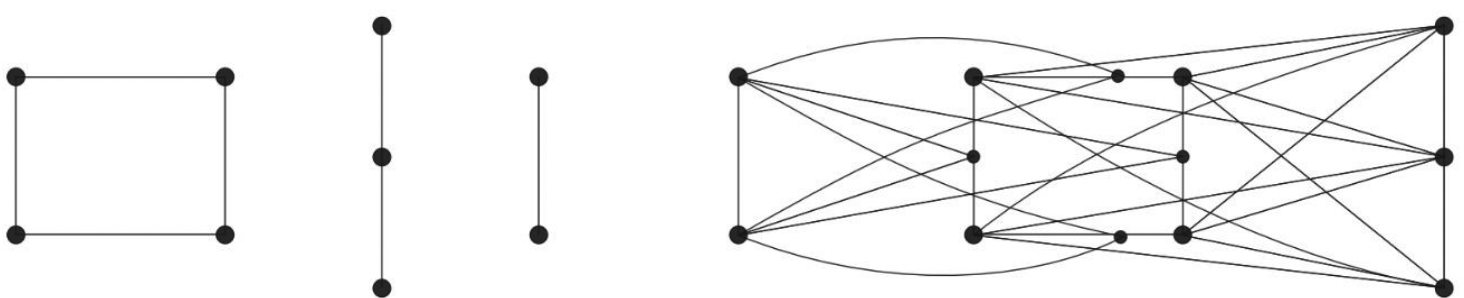

Figure 1: The SVE-join $C_{4}^{\mathcal{S}} \triangleright\left(P_{3}^{\mathcal{V}} \cup K_{2}^{E}\right)$ of graphs.

Khalifeh et al. (2008), Klavžar et al. (1996), Liu et al. (2019), Nagarajan et al. (2014), Pattabiraman and Kandan (2016), Pattabiraman and Paulraja (2012), Siddiqui (2020), Tavakoli and Rahbarnia (2013), Xu et al. (2019), Yang et al. (2019), Yousafei-Azari et al. (2008).

The SVE-join of graphs has been introduced recently in Wen et al. (2019). It is defined as follows. Let $\Gamma_{1}, \Gamma_{2}$, and $\Gamma_{3}$ are three graphs. Let $\delta\left(\Gamma_{1}\right)$ denotes the subdivision of of $\Gamma_{1}$. Then the vertex set of $\Gamma_{1}$ has two portions, one $V\left(\Gamma_{1}\right)$ that contains the original vertices of the graph $\Gamma_{1}$, and the other is denoted by $V^{\prime}\left(\Gamma_{1}\right)$, which includes the new vertices that are inserted into the old edges to subdivide them the edges of $\Gamma_{1}$. Let $\Gamma_{1}^{\mathcal{S}} \triangleright\left(\Gamma_{2}^{\mathcal{V}} \cup \Gamma_{3}^{E}\right)$ denotes the SVE-join of our graphs $\Gamma_{1}, \Gamma_{2}$ and $\Gamma_{3}$. The vertex set of

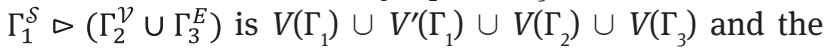
edge set of $\Gamma_{1}^{\mathcal{S}} \triangleright\left(\Gamma_{2}^{\mathcal{V}} \cup \Gamma_{3}^{E}\right)$ is the set $E\left(\Gamma_{1}\right) \cup E\left(\Gamma_{2}\right) \cup E\left(\Gamma_{3}\right)$ and all edges that join the vertices of $\Gamma_{1}$ with the vertices of $\Gamma_{3}$ and all edges joining the vertices in $V^{\prime}\left(\Gamma_{1}\right)$ with all the vertices of $\Gamma_{2}$.

Consider three graphs $C_{4}, P_{3}$, and $K_{2}$. Then the SVE join $C_{4}^{\mathcal{S}} \triangleright\left(P_{3}^{\mathcal{V}} \cup K_{2}^{E}\right)$ is shown in Figure 1. It can bee seen that the edge subdivision of $C_{4}$ is performed first and then the vertices of $C_{4}$ are joined with all of $P_{3}$ and the new vertices introduced in the subdivision of $C_{4}$ are joined with all vertices of $K_{2}$.

\section{Previous results}

For an edge $x y$ of the graph $\Gamma$, let $N_{\Gamma}(x y)$ be the set of common neighbors of $x$ and $y$, and $\mathrm{N}_{\Gamma}^{\prime}(x y)$ be a set of edges that are lying at distance one from $x$ and $y$. The set $\mathrm{N}_{\Gamma}^{\prime}(x)\left(\mathrm{N}_{\Gamma}(y)\right)$ have the edges that are at distance one from $x(y)$.

In the following lemma, we present some structural properties of the SVE-join of graphs.

\section{Lemma 5.1}

According to Wen et al. (2019), let $\Gamma_{1}, \Gamma_{2}$ and $\Gamma_{3}$ be graphs. Then the degree of vertices in the SVE join $\Gamma_{1}^{\mathcal{S}} \triangleright\left(\Gamma_{2}^{\mathcal{V}} \cup \Gamma_{3}^{\mathrm{E}}\right)$ and the number of vertices and edges closer to arbitrary vertices and edges in this product is given below:

1. $\left|V\left(\Gamma_{1}^{\mathcal{S}} \triangleright\left(\Gamma_{2}^{\mathcal{V}} \cup \Gamma_{3}^{E}\right)\right)\right|=n_{1}+m_{1}+n_{2}+n_{3}$ and $\left|E\left(\Gamma_{1}^{\mathcal{S}} \triangleright\left(\Gamma_{2}^{\mathcal{V}} \cup \Gamma_{3}^{E}\right)\right)\right|=2 m_{1}+n_{1} n_{2}+m_{1} n_{3}+m_{2}+m_{3}$.

2.

$$
d_{\Gamma_{1}^{S} \triangleright\left(\Gamma_{2}^{v} \cup \Gamma_{3}^{E}\right)}(x)= \begin{cases}d_{\Gamma_{1}}(x)+n_{2}, & \text { if } x \in V\left(\Gamma_{1}\right), \\ n_{3}+2, & \text { if } x \in E\left(\Gamma_{1}\right), \\ d_{\Gamma_{2}}(x)+n_{1}, & \text { if } x \in V\left(\Gamma_{2}\right), \\ d_{\Gamma_{3}}(x)+m_{1}, & \text { if } x \in V\left(\Gamma_{3}\right) .\end{cases}
$$

3.

$$
\mathrm{n}_{x}\left(e=x y \mid\left(\Gamma_{1}^{\mathcal{S}} \triangleright\left(\Gamma_{2}^{\mathcal{V}} \cup \Gamma_{3}^{E}\right)\right)= \begin{cases}d_{\Gamma_{2}}(x)-\left|N_{\Gamma_{2}}(x y)\right|, & \text { if } x y \in E\left(\Gamma_{2}\right), \\ d_{\Gamma_{3}}(x)-\left|N_{\Gamma_{3}}(x y)\right|, & \text { if } x y \in E\left(\Gamma_{3}\right), \\ n_{2}+n_{3}-d_{\Gamma_{2}}(y)+d_{\Gamma_{1}}(x), & \text { if } x \in V\left(\Gamma_{1}\right) \text { and } y \in V\left(\Gamma_{2}\right), \\ n_{2}+n_{3}+2-d_{\Gamma_{3}}(y), & \text { if } x \in E\left(\Gamma_{1}\right) \text { and } y \in V\left(\Gamma_{3}\right), \\ n_{2}+n_{1}-2+d_{\Gamma_{1}}(x), & \text { if } x \in V\left(\Gamma_{1}\right) \text { and } y \in E\left(\Gamma_{1}\right) .\end{cases}\right.
$$

4.

$$
\mathrm{n}_{y}\left(e=x y \mid\left(\Gamma_{1}^{\mathcal{S}} \triangleright\left(\Gamma_{2}^{\mathcal{V}} \cup \Gamma_{3}^{E}\right)\right)= \begin{cases}d_{\Gamma_{2}}(y)-\left|N_{\Gamma_{2}}(x y)\right|, & \text { if } x y \in E\left(\Gamma_{2}\right), \\ d_{\Gamma_{3}}(y)-\left|N_{\Gamma_{3}}(x y)\right|, & \text { if } x y \in E\left(\Gamma_{3}\right), \\ n_{1}+m_{1}-d_{\Gamma_{1}}(x), & \text { if } x \in V\left(\Gamma_{1}\right) \text { and } y \in V\left(\Gamma_{2}\right), \\ n_{1}+m_{1}-2, & \text { if } x \in E\left(\Gamma_{1}\right) \text { and } y \in V\left(\Gamma_{3}\right), \\ m_{1}+n_{3}+2-d_{\Gamma_{1}}(x), & \text { if } x \in V\left(\Gamma_{1}\right) \text { and } y \in E\left(\Gamma_{1}\right) .\end{cases}\right.
$$


5.

$$
\mathrm{m}_{x}\left(e=x y \mid\left(\Gamma_{1}^{\mathcal{S}} \triangleright\left(\Gamma_{2}^{\mathcal{V}} \cup \Gamma_{3}^{E}\right)\right)=\left\{\begin{array}{l}
n_{1}+\left|N_{\Gamma_{2}}^{\prime}\left(x_{2}\right)\right|-\left|N_{\Gamma_{2}}^{\prime}\left(x_{2} x_{2}^{\prime}\right)\right| \\
+d_{\Gamma_{2}}\left(x_{2}\right)-d_{\Gamma_{2}}\left(x_{2}^{\prime}\right), \\
m_{1}+\left|N_{\Gamma_{3}}^{\prime}\left(x_{3}\right)\right|-\left|N_{\Gamma_{3}}^{\prime}\left(x_{3} x_{3}^{\prime}\right)\right| \\
+d_{\Gamma_{3}}\left(x_{3}\right)-d_{\Gamma_{3}}\left(x_{3}^{\prime}\right), \\
m_{3}+n_{2}-1+m_{2}-\left|N_{\Gamma_{2}}^{\prime}\left(x_{2}\right)\right| \\
+\left(n_{3}+1\right) d_{\Gamma_{1}}\left(x_{1}\right)-d_{\Gamma_{2}}\left(x_{2}\right),
\end{array} \quad \text { if } x y \in E\left(\Gamma_{2}\right),\right.\right.
$$

6.

$$
\mathrm{m}_{y}\left(e=x y \mid\left(\Gamma_{1}^{\mathcal{S}} \triangleright\left(\Gamma_{2}^{\mathcal{V}} \cup \Gamma_{3}^{E}\right)\right)= \begin{cases}n_{1}+\left|N_{\Gamma_{2}}^{\prime}\left(x_{2}^{\prime}\right)\right|-\left|N_{\Gamma_{2}}^{\prime}\left(x_{2} x_{2}^{\prime}\right)\right| & \\ +d_{\Gamma_{2}}\left(x_{2}^{\prime}\right)-d_{\Gamma_{2}}\left(x_{2}\right), & \text { if } x y \in E\left(\Gamma_{2}\right), \\ m_{1}+\left|N_{\Gamma_{3}}^{\prime}\left(x_{3}^{\prime}\right)\right|-\left|N_{\Gamma_{3}}^{\prime}\left(x_{3} x_{3}^{\prime}\right)\right| & \\ +d_{\Gamma_{3}}\left(x_{3}^{\prime}\right)-d_{\Gamma_{3}}\left(x_{3}\right), & \text { if } x y \in E\left(\Gamma_{3}\right), \\ 2 m_{1}+n_{1}-1-2 d_{\Gamma_{1}}\left(x_{1}\right) & \text { if } x \in V\left(\Gamma_{1}\right) \text { and } y \in V\left(\Gamma_{2}\right), \\ +d_{\Gamma_{2}}\left(x_{2}\right), & \\ 3 m_{1}-1-\left(d_{\Gamma_{1}}\left(x_{1}\right)\right. & \text { if } x \in E\left(\Gamma_{1}\right) \text { and } y \in V\left(\Gamma_{3}\right), \\ \left.+d_{\Gamma_{1}}\left(x_{1}^{\prime}\right)\right)+d_{\Gamma_{3}}\left(x_{3}\right), & \text { if } x \in V\left(\Gamma_{1}\right) \text { and } y=x z \in E\left(\Gamma_{1}\right) . \\ m_{3}+m_{1} n_{3}+d_{\Gamma_{1}}\left(x_{1}^{\prime}\right), & \end{cases}\right.
$$

\section{Main results}

Now we proceed towards the main calculations of several Szeged and PI-type indices of SVE join of graphs. First we

calculate the vertex PI and vertex Szeged indices of SVE join of graphs.

\subsection{Vertex Padmakar-Ivan and vertex Szeged indices of SVE}

\section{Theorem 6.1}

Let $\Gamma_{1}, \Gamma_{2}$, and $\Gamma_{3}$ be three graphs. Then:

$$
\begin{aligned}
P I_{v}\left(\Gamma_{1}^{\mathrm{S}} \triangleright\left(\Gamma_{2}^{\mathrm{V}} \cup \Gamma_{3}^{\mathrm{I}}\right)\right) & =M_{1}\left(\Gamma_{2}\right)+M_{1}\left(\Gamma_{3}\right)-2 m_{2}\left|N_{\Gamma_{2}}\left(x_{2} x_{2}^{\prime}\right)\right|-2 m_{3}\left|N_{\Gamma_{3}}\left(x_{3} x^{\prime}{ }_{3}\right)\right| \\
& +\left(n_{1} n_{2}+n_{3} m_{1}+n_{1} m_{1}\right)\left(n_{1}+n_{2}+n_{3}+m_{1}\right)-2 n_{1} m_{2}-2 m_{1} m_{3} .
\end{aligned}
$$

Proof.

$$
\begin{aligned}
P I_{v}\left(\Gamma_{1}^{\mathrm{S} \triangleright}\left(\Gamma_{2}^{\mathrm{V}} \cup \Gamma_{3}^{\mathrm{I}}\right)\right) & =\sum_{u v \in E\left(\Gamma_{2}\right)}\left(d_{\Gamma_{2}}\left(x_{2}\right)-\left|N_{\Gamma_{2}}\left(x_{2} x_{2}^{\prime}\right)\right|+d_{\Gamma_{2}}\left(x_{2}^{\prime}\right)-\left|N_{\Gamma_{2}}\left(x_{2} x_{2}^{\prime}\right)\right|\right) \\
& +\sum_{u v \in E\left(\Gamma_{3}\right)}\left(d_{\Gamma_{3}}\left(x_{3}\right)-\left|N_{\Gamma_{3}}\left(x_{3} x^{\prime}{ }_{3}\right)\right|+d_{\Gamma_{3}}\left(x_{3}^{\prime}\right)-\left|N_{\Gamma_{3}}\left(x_{3} x_{3}^{\prime}\right)\right|\right) \\
& +\sum_{x_{1} \in V\left(\Gamma_{1}\right)} \sum_{x_{2} \in V\left(\Gamma_{2}\right)}\left(n_{2}-d_{\Gamma_{2}}\left(x_{2}\right)+n_{3}+d_{\Gamma_{1}}\left(x_{1}\right)+n_{1}-d_{\Gamma_{1}}\left(x_{1}\right)+m_{1}\right) \\
& +\sum_{x_{1} \in E\left(\Gamma_{1}\right)} \sum_{x_{3} \in V\left(\Gamma_{3}\right)}\left(n_{3}-d_{\Gamma_{3}}\left(x_{3}\right)+2+n_{2}+n_{1}+m_{1}-2\right) \\
& +\sum_{x_{1} \in V\left(\Gamma_{1}\right)} \sum_{x \prime_{1} \in E\left(\Gamma_{1}\right)}\left(n_{2}+n_{1}-2+d_{\Gamma_{1}}\left(x_{1}\right)+m_{1}+n_{3}+2-d_{\Gamma_{1}}\left(x_{1}\right)\right) \\
& =\sum_{u v \in E\left(\Gamma_{2}\right)}\left(d_{\Gamma_{2}}\left(x_{2}\right)+d_{\Gamma_{2}}\left(x_{2}^{\prime}\right)\right)-2 \sum_{u v \in E\left(\Gamma_{2}\right)}\left|N_{\Gamma_{2}}\left(x_{2} x_{2}^{\prime}{ }_{2}\right)\right| \\
& +\sum_{u v \in E\left(\Gamma_{3}\right)}\left(d_{\Gamma_{3}}\left(x_{3}\right)+d_{\Gamma_{3}}\left(x_{3}^{\prime}\right)\right)-2 \sum_{u v \in E\left(\Gamma_{3}\right)}\left|N_{\Gamma_{3}}\left(x_{3} x_{3}^{\prime}\right)\right| \\
& +n_{1} n_{2}\left(n_{1}+n_{2}+n_{3}+m_{1}\right)-n_{1} \sum_{x_{2} \in V\left(\Gamma_{2}\right)} d_{\Gamma_{2}}\left(x_{2}\right)+m_{1} n_{3}\left(n_{1}+n_{2}+n_{3}+m_{1}\right) \\
& -m_{1} \sum_{x_{3} \in V\left(\Gamma_{2}\right)} \omega \Gamma_{2}\left(x_{3}\right)+n_{1} m_{1}\left(n_{1}+n_{2}+n_{3}+m_{1}\right) \\
& =M_{1}\left(\Gamma_{2}\right)+M_{1}\left(\Gamma_{3}\right)-2 m_{2}\left|N_{\Gamma_{2}}\left(x_{2} x_{2}^{\prime}\right)\right|-2 m_{3}\left|N_{\Gamma_{3}}\left(x_{3} x_{3}^{\prime}{ }_{3}\right)\right| \\
& +\left(n_{1} n_{2}+n_{3} m_{1}+n_{1} m_{1}\right)\left(n_{1}+n_{2}+n_{3}+m_{1}\right)-2 n_{1} m_{2}-2 m_{1} m_{3} .
\end{aligned}
$$

This completes the proof. 


\section{Theorem 6.2}

Let $\Gamma_{1}, \Gamma_{2}$, and $\Gamma_{3}$ be three graphs. Then:

$$
\begin{aligned}
S z_{v}\left(\Gamma_{1}^{\mathrm{S}} \triangleright\left(\Gamma_{2}^{\mathrm{V}} \cup \Gamma_{3}^{\mathrm{I}}\right)\right) & =M_{2}\left(\Gamma_{2}\right)+M_{2}\left(\Gamma_{3}\right)-\left|N_{\Gamma_{2}}\left(x_{2} x_{2}^{\prime}\right)\right| M_{1}\left(\Gamma_{2}\right)-\left|N_{\Gamma_{3}}\left(x_{3} x^{\prime}{ }_{3}\right)\right| M_{1}\left(\Gamma_{3}\right) \\
& -\left(n_{2}+m_{1}\right) M_{1}\left(\Gamma_{1}\right)+m_{2}\left|N_{\Gamma_{2}}\left(x_{2} x^{\prime}{ }_{2}\right)\right|^{2}+m_{3}\left|N_{\Gamma_{3}}\left(x_{3} x_{3}^{\prime}\right)\right|^{2}+n_{1} n_{2}\left(n_{2}+n_{3}\right) \\
& \left(n_{1}+m_{1}\right)+2 m_{1} n_{2}\left(n_{1}+m_{1}-n_{2}-n_{3}\right)-2 n_{1} m_{2}\left(n_{1}+m_{1}\right)+4 m_{1} m_{2}+ \\
& m_{1} n_{3}\left(n_{2}+n_{3}+2\right)\left(m_{1}+n_{1}-2\right)-2 m_{1} m_{3}\left(n_{1}+m_{1}-2\right) \\
& +n_{1} m_{1}\left(n_{1}+n_{2}-2\right)\left(n_{3}+m_{1}+2\right)+2 m_{1}^{2}\left(n_{2}+n_{1}-m_{1}-n_{3}-4\right) .
\end{aligned}
$$

Proof.

$$
\begin{aligned}
& S z_{v}\left(\Gamma_{1}^{\mathrm{S}} \triangleright\left(\Gamma_{2}^{\mathrm{V}} \cup \Gamma_{3}^{\mathrm{I}}\right)\right)=\sum_{u v \in E\left(\Gamma_{2}\right)}\left(d_{\Gamma_{2}}\left(x_{2}\right)-\left|N_{\Gamma_{2}}\left(x_{2} x_{2}^{\prime}\right)\right|\right)\left(d_{\Gamma_{2}}\left(x^{\prime}{ }_{2}\right)-\left|N_{\Gamma_{2}}\left(x_{2} x_{2}^{\prime}\right)\right|\right) \\
& +\sum_{u v \in E\left(\Gamma_{3}\right)}\left(d_{\Gamma_{3}}\left(x_{3}\right)-\left|N_{\Gamma_{3}}\left(x_{3} x_{3}^{\prime}\right)\right|\right)\left(d_{\Gamma_{3}}\left(x_{3}^{\prime}\right)-\left|N_{\Gamma_{3}}\left(x_{3} x_{3}^{\prime}\right)\right|\right) \\
& +\sum_{x_{1} \in V\left(\Gamma_{1}\right)} \sum_{x_{2} \in V\left(\Gamma_{2}\right)}\left(n_{2}-d_{\Gamma_{2}}\left(x_{2}\right)+n_{3}+d_{\Gamma_{1}}\left(x_{1}\right)\right)\left(n_{1}-d_{\Gamma_{1}}\left(x_{1}\right)+m_{1}\right) \\
& +\sum_{x_{1} \in E\left(\Gamma_{1}\right)} \sum_{x_{3} \in V\left(\Gamma_{3}\right)}\left(n_{3}-d_{\Gamma_{3}}\left(x_{3}\right)+2+n_{2}\right)\left(n_{1}+m_{1}-2\right) \\
& +\sum_{x_{1} \in V\left(\Gamma_{1}\right)} \sum_{x^{\prime} \in E\left(\Gamma_{1}\right)}\left(n_{2}+n_{1}-2+d_{\Gamma_{1}}\left(x_{1}\right)\right)\left(m_{1}+n_{3}+2-d_{\Gamma_{1}}\left(x_{1}\right)\right) \\
& =\sum_{u v \in E\left(\Gamma_{2}\right)}\left(d_{\Gamma_{2}}\left(x_{2}\right) d_{\Gamma_{2}}\left(x_{2}^{\prime}\right)-\left|N_{\Gamma_{2}}\left(x_{2} x_{2}^{\prime}\right)\right|\left(d_{\Gamma_{2}}\left(x_{2}\right)+d_{\Gamma_{2}}\left(x_{2}^{\prime}\right)\right)\right. \\
& \left.+\left|N_{\Gamma_{2}}\left(x_{2} x_{2}^{\prime}\right)\right|^{2}\right)+\sum_{u v \in E\left(\Gamma_{3}\right)}\left(d_{\Gamma_{3}}\left(x_{3}\right) d_{\Gamma_{3}}\left(x_{3}^{\prime}\right)-\left|N_{\Gamma_{3}}\left(x_{3} x_{3}^{\prime}\right)\right|\left(d_{\Gamma_{3}}\left(x_{3}\right)\right.\right. \\
& \left.\left.+d_{\Gamma_{3}}\left(x_{3}^{\prime}\right)\right)+\left|N_{\Gamma_{3}}\left(x_{3} x^{\prime}{ }_{3}\right)\right|^{2}\right)+\sum_{x_{1} \in V\left(\Gamma_{1}\right)} \sum_{x_{2} \in V\left(\Gamma_{2}\right)}\left(\left(n_{2}+n_{3}\right)\left(n_{1}+m_{1}\right)\right. \\
& +\left(n_{1}+m_{1}-n_{2}-n_{3}\right) d_{\Gamma_{1}}\left(x_{1}^{\prime}\right)-\left(n_{1}+m_{1}\right) d_{\Gamma_{2}}\left(x_{2}^{\prime}\right)-\omega_{\Gamma_{1}}^{2}\left(x_{1}^{\prime}\right) \\
& +d_{\Gamma_{1}}\left(x_{1}^{\prime}\right) d_{\Gamma_{2}}\left(x_{2}^{\prime}\right)+\sum_{x_{1} \in E\left(\Gamma_{1}\right)} \sum_{x_{3} \in V\left(\Gamma_{3}\right)}\left(n_{1}+m_{1}-2\right)\left(n_{3}+n_{2}+2\right) \\
& \left.-\left(n_{1}+m_{1}-2\right) \sum_{x_{1} \in E\left(\Gamma_{1}\right)} \sum_{x_{3} \in V\left(\Gamma_{3}\right)} d_{\Gamma_{3}}\left(x_{3}\right)\right) \\
& +\sum_{x_{1} \in V\left(\Gamma_{1}\right)} \sum_{x_{\prime_{1} \in E\left(\Gamma_{1}\right)}}\left(\left(n_{2}+n_{1}-2\right)\left(m_{1}+n_{3}+2\right)-\omega_{\Gamma_{1}}^{2}\left(x_{1}\right)\right. \\
& \left.-\left(n_{2}+n_{1}-m_{1}-n_{3}-4\right) d_{\Gamma_{1}}\left(x_{1}\right)\right) \\
& =M_{2}\left(\Gamma_{2}\right)-\left|N_{\Gamma_{2}}\left(x_{2} x_{2}^{\prime}\right)\right| M_{1}\left(\Gamma_{2}\right)+m_{2}\left|N_{\Gamma_{2}}\left(x_{2} x_{2}^{\prime}\right)\right|^{2}+M_{2}\left(\Gamma_{3}\right) \\
& -\left|N_{\Gamma_{3}}\left(x_{3} x^{\prime}{ }_{3}\right)\right| M_{1}\left(\Gamma_{3}\right)+m_{3}\left|N_{\Gamma_{3}}\left(x_{3} x_{3}^{\prime}\right)\right|^{2}+n_{1} n_{2}\left(n_{2}+n_{3}\right)\left(n_{1}+m_{1}\right) \\
& +2 m_{1} n_{2}\left(n_{1}+m_{1}-n_{2}-n_{3}\right)-2 n_{1} m_{2}\left(n_{1}+m_{1}\right)-n_{2} M_{1}\left(\Gamma_{1}\right)+4 m_{1} m_{2} \\
& +m_{1} n_{3}\left(n_{2}+n_{3}+2\right)\left(m_{1}+n_{1}-2\right)-2 m_{1} m_{3}\left(n_{1}+m_{1}-2\right)+n_{1} m_{1}\left(n_{1}+n_{2}-2\right) \\
& \left(n_{3}+m_{1}+2\right)-m_{1} M_{1}\left(\Gamma_{1}\right)+2 m_{1}^{2}\left(n_{2}+n_{1}-m_{1}-n_{3}-4\right) \\
& =M_{2}\left(\Gamma_{2}\right)+M_{2}\left(\Gamma_{3}\right)-\left|N_{\Gamma_{2}}\left(x_{2} x_{2}^{\prime}\right)\right| M_{1}\left(\Gamma_{2}\right)-\left|N_{\Gamma_{3}}\left(x_{3} x_{3}^{\prime}\right)\right| M_{1}\left(\Gamma_{3}\right) \\
& -\left(n_{2}+m_{1}\right) M_{1}\left(\Gamma_{1}\right)+m_{2}\left|N_{\Gamma_{2}}\left(x_{2} x_{2}^{\prime}\right)\right|^{2}+m_{3}\left|N_{\Gamma_{3}}\left(x_{3} x_{3}^{\prime}\right)\right|^{2}+n_{1} n_{2}\left(n_{2}+n_{3}\right) \\
& \left(n_{1}+m_{1}\right)+2 m_{1} n_{2}\left(n_{1}+m_{1}-n_{2}-n_{3}\right)-2 n_{1} m_{2}\left(n_{1}+m_{1}\right)+4 m_{1} m_{2}+m_{1} n_{3}\left(n_{2}+n_{3}+2\right) \\
& \left(m_{1}+n_{1}-2\right)-2 m_{1} m_{3}\left(n_{1}+m_{1}-2\right)+n_{1} m_{1}\left(n_{1}+n_{2}-2\right)\left(n_{3}+m_{1}+2\right) \\
& +2 m_{1}^{2}\left(n_{2}+n_{1}-m_{1}-n_{3}-4\right) \text {. }
\end{aligned}
$$

This completes the proof.

\subsection{Edge Padmakar-Ivan and edge Szeged indices}

In this section, we calculate the edge PI and edge Szeged indices of SVE join of graphs.

\section{Theorem 6.3}

Let $\Gamma_{1}, \Gamma_{2}$, and $\Gamma_{3}$ be three graphs. Then:

$$
\begin{aligned}
P I_{e}\left(\Gamma_{1}^{\mathrm{S}} \triangleright\left(\Gamma_{2}^{\mathrm{V}} \cup \Gamma_{3}^{\mathrm{I}}\right)\right) & =2 m_{2}\left(2 n_{1}+\mid N_{\Gamma_{2}}^{\prime}\left(x_{2}\right)\right)+2 m_{3}\left(2 \epsilon_{1}+\left|N_{\Gamma_{3}}^{\prime}\left(x_{3}\right)\right|\right)-4 m_{2}\left|N_{\Gamma_{2}}^{\prime}\left(x_{2} x_{2}^{\prime}\right)\right| \\
& -4 m_{3}\left|N_{\Gamma_{3}}^{\prime}\left(x_{3} x_{3}^{\prime}\right)\right|+\left(2 m_{1}+n_{1} n_{2}\right)\left|N_{\Gamma_{2}}^{\prime}\left(x_{2}^{\prime}\right)\right|+\left(2 m_{3}-m_{1} n_{2}\right)\left|N_{\Gamma_{3}}^{\prime}\left(x_{3}^{\prime}\right)\right| \\
& +n_{1} n_{2}\left(m_{3}+n_{2}+m_{2}+2 m_{1}+n_{1}-2\right)+m_{1} n_{2}\left(2 n_{2}+m_{2}+3 n_{3}+m_{3}+3 m_{1}-2\right) \\
& +\left(n_{1}-n_{2}\right) M_{1}\left(\Gamma_{1}\right)+n_{1} m_{1}\left(m_{2}+n_{1} n_{2}-n_{2}+m_{3}+m_{1} n_{3}-2\right)+4 m_{1}^{2} .
\end{aligned}
$$


Proof.

$$
\begin{aligned}
& P I_{e}\left(\Gamma_{1}^{\mathrm{S}} \triangleright\left(\Gamma_{2}^{\mathrm{V}} \cup \Gamma_{3}^{\mathrm{I}}\right)\right)=\sum_{u v \in E\left(\Gamma_{2}\right)}\left(n_{1}+\left|N_{\Gamma_{2}}^{\prime}\left(x_{2}\right)\right|-\left|N_{\Gamma_{2}}^{\prime}\left(x_{2} x_{2}^{\prime}\right)\right|+d_{\Gamma_{2}}\left(x_{2}\right)-d_{\Gamma_{2}}\left(x_{2}^{\prime}\right)\right. \\
& \left.+n_{1}+\left|N_{\Gamma_{2}}^{\prime}\left(x_{2}^{\prime}\right)\right|-\left|N_{\Gamma_{2}}^{\prime}\left(x_{2} x_{2}^{\prime}\right)\right|+d_{\Gamma_{2}}\left(x_{2}^{\prime}\right)-d_{\Gamma_{2}}\left(x_{2}\right)\right) \\
& +\sum_{u v \in E\left(\Gamma_{3}\right)}\left(m_{1}+\left|N_{\Gamma_{3}}^{\prime}\left(x_{3}\right)\right|-\left|N_{\Gamma_{3}}^{\prime}\left(x_{3} x_{3}^{\prime}\right)\right|+d_{\Gamma_{3}}\left(x_{3}\right)-d_{\Gamma_{3}}\left(x_{3}^{\prime}\right)\right. \\
& \left.+m_{1}+\left|N_{\Gamma_{3}}^{\prime}\left(x_{3}^{\prime}\right)\right|-\left|N_{\Gamma_{3}}^{\prime}\left(x_{3} x^{\prime}{ }_{3}\right)\right|+d_{\Gamma_{3}}\left(x^{\prime}{ }_{3}\right)-d_{\Gamma_{3}}\left(x_{3}\right)\right) \\
& +\sum_{x_{1} \in V\left(\Gamma_{1}\right)} \sum_{x_{2} \in V\left(\Gamma_{2}\right)}\left(m_{3}+n_{2}-1+m_{2}-\left|N_{\Gamma_{2}}^{\prime}\left(x_{2}\right)\right|+\left(n_{3}+1\right) d_{\Gamma_{1}}\left(x_{1}\right)\right. \\
& \left.-d_{\Gamma_{2}}\left(x_{2}\right)+2 m_{1}+n_{1}-1-2 d_{\Gamma_{1}}\left(x_{1}\right)+d_{\Gamma_{2}}\left(x_{2}\right)\right) \\
& +\sum_{x_{1} \in E\left(\Gamma_{1}\right)} \sum_{x_{3} \in V\left(\Gamma_{3}\right)}\left(2 n_{2}+m_{2}+n_{3}+m_{3}+1-\left|N_{\Gamma_{3}}^{\prime}\left(x_{3}\right)\right|-d_{\Gamma_{3}}\left(x_{3}\right)\right. \\
& +3 m_{1}-1-\left(d_{\Gamma_{1}}\left(x_{1}\right)+d_{\Gamma_{1}}\left(x_{1}^{\prime}\right)\right)+d_{\Gamma_{3}}\left(x_{3}\right) \\
& +\sum_{x_{1} \in V\left(\Gamma_{1}\right)} \sum_{x_{1} x_{1} \in E\left(\Gamma_{1}\right)}\left(m_{2}+n_{1} n_{2}-n_{2}-2+2 d_{\Gamma_{1}}\left(x_{1}\right)+m_{3}+m_{1} n_{3}+d_{\Gamma_{1}}\left(x_{1}^{\prime}\right)\right) \\
& =2 m_{2}\left(2 n_{1}+\left|N_{\Gamma_{2}}^{\prime}\left(x_{2}\right)+\right| N_{\Gamma_{2}}^{\prime}\left(x_{2}\right)|-2| N_{\Gamma_{2}}^{\prime}\left(x_{2} x^{\prime}{ }_{2}\right) \mid\right)+2 m_{3}\left(2 \epsilon_{1}+\left|N_{\Gamma_{3}}^{\prime}\left(x_{3}\right)\right|\right. \\
& \left.+\left|N_{\Gamma_{3}}^{\prime}\left(x_{3}\right)\right|-2\left|N_{\Gamma_{3}}^{\prime}\left(x_{3} x_{3}^{\prime}\right)\right|\right)+n_{1} n_{2}\left(m_{3}+n_{2}+m_{2}+2 m_{1}+n_{1}-2-\left|N_{\Gamma_{2}}^{\prime}\left(x_{2}\right)\right|\right) \\
& +\sum_{x_{1} \in V\left(\Gamma_{1}\right)} \sum_{x_{2} \in V\left(\Gamma_{2}\right)}\left(n_{3}-1\right) d_{\Gamma_{1}}\left(x_{1}\right)+m_{1} n_{2}\left(2 n_{2}+m_{2}+n_{3}+m_{3}+3 m_{1}\right. \\
& \left.-\left|N_{\Gamma_{3}}^{\prime}\left(x_{3}\right)\right|\right)-\sum_{x_{1} \in E\left(\Gamma_{1}\right)} \sum_{x_{3} \in V\left(\Gamma_{3}\right)}\left(d_{\Gamma_{1}}\left(x_{1}\right)+d_{\Gamma_{1}}\left(x_{1}^{\prime}\right)\right)+n_{1} m_{1}\left(m_{2}+n_{1} n_{2}\right. \\
& \left.-n_{2}+m_{3}+m_{1} n_{3}-2\right)+\sum_{x_{1} \in V\left(\Gamma_{1}\right)} \sum_{x_{1} \in E\left(\Gamma_{1}\right)} d_{\Gamma_{1}}\left(x_{1}\right) \\
& +\sum_{x_{1} \in V\left(\Gamma_{1}\right)} \sum_{x_{1} x_{1} \in E\left(\Gamma_{1}\right)}\left(d_{\Gamma_{1}}\left(x_{1}\right)+d_{\Gamma_{1}}\left(x_{1}\right)\right) \\
& =2 m_{2}\left(2 n_{1}+\mid N_{\Gamma_{2}}^{\prime}\left(x_{2}\right)\right)+2 m_{3}\left(2 \epsilon_{1}+\left|N_{\Gamma_{3}}^{\prime}\left(x_{3}\right)\right|\right)-4 m_{2}\left|N_{\Gamma_{2}}^{\prime}\left(x_{2} x_{2}^{\prime}{ }_{2}\right)\right| \\
& -4 m_{3}\left|N_{\Gamma_{3}}^{\prime}\left(x_{3} x^{\prime}{ }_{3}\right)\right|+\left(2 m_{1}+n_{1} n_{2}\right)\left|N_{\Gamma_{2}}^{\prime}\left(x_{2}^{\prime}\right)\right|+\left(2 m_{3}-m_{1} n_{2}\right)\left|N_{\Gamma_{3}}^{\prime}\left(x_{3}^{\prime}\right)\right| \\
& +n_{1} n_{2}\left(m_{3}+n_{2}+m_{2}+2 m_{1}+n_{1}-2\right)+m_{1} n_{2}\left(2 n_{2}+m_{2}+3 n_{3}+m_{3}+3 m_{1}-2\right) \\
& +\left(n_{1}-n_{2}\right) M_{1}\left(\Gamma_{1}\right)+n_{1} m_{1}\left(m_{2}+n_{1} n_{2}-n_{2}+m_{3}+m_{1} n_{3}-2\right)+4 m_{1}^{2} \text {. }
\end{aligned}
$$

This completes the proof.

\section{Theorem 6.4}

Let $\Gamma_{1}, \Gamma_{2}$, and $\Gamma_{3}$ be three graphs. Then:

$$
\begin{aligned}
& S z_{e}\left(\Gamma_{1}^{\mathrm{S}} \triangleright\left(\Gamma_{2}^{\mathrm{V}} \cup \Gamma_{3}^{\mathrm{I}}\right)\right)=2\left(M_{1}\left(\Gamma_{2}\right)+M_{1}\left(\Gamma_{3}\right)\right)-F\left(\Gamma_{2}\right)-F\left(\Gamma_{3}\right)+m_{2}\left(n_{1}+\left|N_{\Gamma_{2}}^{\prime}\left(x_{2}\right)\right|\right. \\
& \left.-\left|N_{\Gamma_{2}}^{\prime}\left(x_{2} x_{2}^{\prime}\right)\right|\right)\left(n_{1}+\left|N_{\Gamma_{2}}^{\prime}\left(x_{2}^{\prime}\right)\right|-\left|N_{\Gamma_{2}}^{\prime}\left(x_{2} x_{2}^{\prime}\right)\right|\right)+m_{3}\left(m_{1}+\left|N_{\Gamma_{3}}^{\prime}\left(x_{3}\right)\right|\right. \\
& \left.-\left|N_{\Gamma_{3}}^{\prime}\left(x_{3} x_{3}^{\prime}\right)\right|\right)\left(m_{1}+\left|N_{\Gamma_{3}}^{\prime}\left(x_{3}^{\prime}\right)\right|-\left|N_{\Gamma_{3}}^{\prime}\left(x_{3} x_{3}^{\prime}\right)\right|\right)+n_{1} n_{2}\left(m_{3}+n_{2}-1+m_{2}\right. \\
& \left.-\left|N_{\Gamma_{2}}^{\prime}\left(x_{2}\right)\right|\right)\left(2 m_{1}+n_{1}-1\right)+2 m_{1} n_{2}\left(\left(n_{3}+1\right)\left(2 m_{1}+n_{1}-1\right)-2\left(m_{3}+n_{2}-1+m_{2}\right.\right. \\
& \left.\left.-\left|N_{\Gamma_{2}}^{\prime}\left(x_{2}\right)\right|\right)\right)+2 m_{2} n_{1}\left(m_{3}+n_{2}+m_{2}-\left|N_{\Gamma_{2}}^{\prime}\left(x_{2}\right)\right|-2 m_{1}-n_{1}\right)-2 n_{2}\left(n_{3}+1\right) \\
& M_{1}\left(\Gamma_{1}\right)+4 m_{1} m_{2}\left(n_{3}+3\right)-n_{1} M_{1}\left(\Gamma_{2}\right)+m_{1} n_{3}\left(\left(2 n_{2}+m_{2}+n_{3}+m_{3}+1\right.\right. \\
& \left.-\left|N_{\Gamma_{3}}^{\prime}\left(x_{3}\right)\right|\right)\left(3 m_{1}-1\right)-n_{3}\left(2 n_{2}+m_{2}+n_{3}+m_{3}+1-\left|N_{\Gamma_{3}}^{\prime}\left(x_{3}\right)\right|\right) M_{1}\left(\Gamma_{1}\right) \\
& +2 m_{1} m_{3}\left(2 n_{2}+m_{2}+n_{3}+m_{3}-3 m_{1}+2-\left|N_{\Gamma_{3}}^{\prime}\left(x_{3}\right)\right|\right)+2 m_{3} M_{1}\left(\Gamma_{1}\right)-m_{1} M_{1}\left(\Gamma_{3}\right) \\
& +n_{1} m_{1}\left(m_{2}+n_{1} n_{2}-n_{2}-2\right)\left(m_{3}+m_{1} n_{3}\right)+n_{1}\left(m_{2}+n_{1} n_{2}-n_{2}-2\right) \\
& \sum_{x_{1} x_{1} \in E\left(\Gamma_{1}\right)} d_{\Gamma_{1}}\left(x_{1}^{\prime}\right)+4 m_{1}^{2}\left(m_{3}+m_{1} n_{3}\right)+2 n_{1} M_{2}\left(\Gamma_{1}\right) \text {. }
\end{aligned}
$$

Proof.

$$
\begin{aligned}
S z_{e}\left(\Gamma_{1}^{\mathrm{S} \triangleright} \triangleright\left(\Gamma_{2}^{\mathrm{V}} \cup \Gamma_{3}^{\mathrm{I}}\right)\right) & =\sum_{u v \in E\left(\Gamma_{2}\right)}\left(n_{1}+\left|N_{\Gamma_{2}}^{\prime}\left(x_{2}\right)\right|-\left|N_{\Gamma_{2}}^{\prime}\left(x_{2} x_{2}^{\prime}\right)\right|+d_{\Gamma_{2}}\left(x_{2}\right)-d_{\Gamma_{2}}\left(x_{2}^{\prime}\right)\right) \\
& \left(n_{1}+\left|N_{\Gamma_{2}}^{\prime}\left(x_{2}^{\prime}\right)\right|-\left|N_{\Gamma_{2}}^{\prime}\left(x_{2} x_{2}^{\prime}\right)\right|+d_{\Gamma_{2}}\left(x_{2}^{\prime}{ }_{2}\right)-d_{\Gamma_{2}}\left(x_{2}\right)\right) \\
& +\sum_{u v \in E\left(\Gamma_{3}\right)}\left(m_{1}+\left|N_{\Gamma_{3}}^{\prime}\left(x_{3}\right)\right|-\left|N_{\Gamma_{3}}^{\prime}\left(x_{3} x_{3}^{\prime}\right)\right|+d_{\Gamma_{3}}\left(x_{3}\right)-d_{\Gamma_{3}}\left(x_{3}^{\prime}\right)\right) \\
& \left(m_{1}+\left|N_{\Gamma_{3}}^{\prime}\left(x_{3}^{\prime}\right)\right|-\left|N_{\Gamma_{3}}^{\prime}\left(x_{3} x_{3}^{\prime}{ }_{3}\right)\right|+d_{\Gamma_{3}}\left(x_{3}^{\prime}{ }_{3}\right)-d_{\Gamma_{3}}\left(x_{3}\right)\right) \\
& +\sum_{x_{1} \in V\left(\Gamma_{1}\right)} \sum_{x_{2} \in V\left(\Gamma_{2}\right)}\left(m_{3}+n_{2}-1+m_{2}-\left|N_{\Gamma_{2}}^{\prime}\left(x_{2}\right)\right|+\left(n_{3}+1\right) d_{\Gamma_{1}}\left(x_{1}\right)\right. \\
& \left.-d_{\Gamma_{2}}\left(x_{2}\right)\right)\left(2 m_{1}+n_{1}-1-2 d_{\Gamma_{1}}\left(x_{1}\right)+d_{\Gamma_{2}}\left(x_{2}\right)\right)+\sum_{x_{1} \in E\left(\Gamma_{1}\right)} \sum_{x_{3} \in V\left(\Gamma_{3}\right)}\left(2 n_{2}\right. \\
& \left.+m_{2}+n_{3}+m_{3}+1-\left|N_{\Gamma_{3}}\left(x_{3}\right)\right|-d_{\Gamma_{3}}\left(x_{3}\right)\right)\left(3 m_{1}-1-\left(d_{\Gamma_{1}}\left(x_{1}\right)+d_{\Gamma_{1}}\left(x_{1}^{\prime}\right)\right)\right. \\
& +d_{\Gamma_{3}}\left(x_{3}\right)+\sum_{x_{1} \in V\left(\Gamma_{1}\right)} \sum_{x_{1} x 1_{1} \in E\left(\Gamma_{1}\right)}\left(m_{2}+n_{1} n_{2}-n_{2}-2+2 d_{\Gamma_{1}}\left(x_{1}\right)\right) \\
& \left(m_{3}+m_{1} n_{3}+d_{\Gamma_{1}}\left(x_{1}^{\prime}\right)\right)
\end{aligned}
$$




$$
\begin{aligned}
& =\sum_{u v \in E\left(\Gamma_{2}\right)}\left(\left(n_{1}+\left|N_{\Gamma_{2}}^{\prime}\left(x_{2}\right)\right|-\left|N_{\Gamma_{2}}^{\prime}\left(x_{2} x_{2}^{\prime}\right)\right|\right)\left(n_{1}+\left|N_{\Gamma_{2}}^{\prime}\left(x_{2}^{\prime}\right)\right|-\left|N_{\Gamma_{2}}^{\prime}\left(x_{2} x_{2}^{\prime}\right)\right|\right)\right. \\
& \left.+\left(d_{\Gamma_{2}}\left(x_{2}\right)-d_{\Gamma_{2}}\left(x_{2}^{\prime}\right)\right)\left(\left|N_{\Gamma_{2}}^{\prime}\left(x_{2}^{\prime}\right)\right|-\left|N_{\Gamma_{2}}^{\prime}\left(x_{2}\right)\right|\right)-\left(d_{\Gamma_{2}}\left(x_{2}^{\prime}\right)-d_{\Gamma_{2}}\left(x_{2}\right)\right)^{2}\right) \\
& +\sum_{u v \in E\left(\Gamma_{3}\right)}\left(\left(m_{1}+\left|N_{\Gamma_{3}}^{\prime}\left(x_{3}\right)\right|-\left|N_{\Gamma_{3}}^{\prime}\left(x_{3} x_{3}^{\prime}\right)\right|\right)\left(m_{1}+\left|N_{\Gamma_{3}}^{\prime}\left(x_{3}^{\prime}\right)\right|-\left|N_{\Gamma_{3}}^{\prime}\left(x_{3} x_{3}^{\prime}\right)\right|\right)\right. \\
& \left.+\left(d_{\Gamma_{3}}\left(x_{3}\right)-d_{\Gamma_{3}}\left(x_{3}^{\prime}\right)\right)\left(\left|N_{\Gamma_{3}}^{\prime}\left(x_{3}^{\prime}\right)\right|-\left|N_{\Gamma_{3}}^{\prime}\left(x_{3}\right)\right|\right)+\left(d_{\Gamma_{3}}\left(x^{\prime}{ }_{3}\right)-d_{\Gamma_{3}}\left(x_{3}\right)\right)^{2}\right) \\
& +\sum_{x_{1} \in V\left(\Gamma_{1}\right)} \sum_{x_{2} \in V\left(\Gamma_{2}\right)}\left(\left(m_{3}+n_{2}-1+m_{2}-\left|N_{\Gamma_{2}}^{\prime}\left(x_{2}\right)\right|\right)\left(2 m_{1}+n_{1}-1\right)\right. \\
& +\left(\left(n_{3}+1\right)\left(2 m_{1}+n_{1}-1\right)-2\left(m_{3}+n_{2}-1+m_{2}-\left|N_{\Gamma_{2}}^{\prime}\left(x_{2}\right)\right|\right)\right) d_{\Gamma_{1}}\left(x_{1}\right) \\
& +\left(m_{3}+n_{2}+m_{2}-\left|N_{\Gamma_{2}}^{\prime}\left(x_{2}\right)\right|-2 m_{1}-n_{1}\right) d_{\Gamma_{2}}\left(x_{2}\right)-2\left(n_{3}+1\right) \omega_{\Gamma_{1}}^{2}\left(x_{1}\right) \\
& \left.+\left(n_{3}+3\right) d_{\Gamma_{1}}\left(x_{1}\right) d_{\Gamma_{2}}\left(x_{2}\right)-\omega_{\Gamma_{2}}^{2}\left(x_{2}\right)\right)+\sum_{x_{1} \in E\left(\Gamma_{1}\right)} \sum_{x_{3} \in V\left(\Gamma_{3}\right)}\left(\left(2 n_{2}+m_{2}\right.\right. \\
& \left.+n_{3}+m_{3}+1-\left|N_{\Gamma_{3}}^{\prime}\left(x_{3}\right)\right|\right)\left(3 m_{1}-1\right)-\left(2 n_{2}+m_{2}+n_{3}+m_{3}+1-\left|N_{\Gamma_{3}}^{\prime}\left(x_{3}\right)\right|\right) \\
& \left(d_{\Gamma_{1}}\left(x_{1}\right)+d_{\Gamma_{1}}\left(x_{1}^{\prime}\right)\right)+\left(2 n_{2}+m_{2}+n_{3}+m_{3}-3 m_{1}+2-\left|N_{\Gamma_{3}}^{\prime}\left(x_{3}\right)\right|\right) d_{\Gamma_{3}}\left(x_{3}\right) \\
& \left.+d_{\Gamma_{3}}\left(x_{3}\right)\left(d_{\Gamma_{1}}\left(x_{1}\right)+d_{\Gamma_{1}}\left(x_{1}^{\prime}\right)\right)-\omega_{\Gamma_{3}}^{2}\left(x_{3}\right)\right)+\sum_{x_{1} \in V\left(\Gamma_{1}\right)} \sum_{x_{1} x_{1} \in E\left(\Gamma_{1}\right)}\left(\left(m_{2}+n_{1} n_{2}\right.\right. \\
& \left.-n_{2}-2\right)\left(m_{3}+m_{1} n_{3}\right)+\left(m_{2}+n_{1} n_{2}-n_{2}-2\right) d_{\Gamma_{1}}\left(x_{1}^{\prime}\right)+2\left(m_{3}+m_{1} n_{3}\right) d_{\Gamma_{1}}\left(x_{1}\right) \\
& \left.+2 d_{\Gamma_{1}}\left(x_{1}\right) d_{\Gamma_{1}}\left(x_{1}^{\prime}\right)\right) \\
& =2\left(M_{1}\left(\Gamma_{2}\right)+M_{1}\left(\Gamma_{3}\right)\right)-F\left(\Gamma_{2}\right)-F\left(\Gamma_{3}\right)+m_{2}\left(n_{1}+\left|N_{\Gamma_{2}}^{\prime}\left(x_{2}\right)\right|-\left|N_{\Gamma_{2}}^{\prime}\left(x_{2} x_{2}^{\prime}\right)\right|\right) \\
& \left(n_{1}+\left|N_{\Gamma_{2}}^{\prime}\left(x_{2}^{\prime}{ }_{2}\right)\right|-\left|N_{\Gamma_{2}}^{\prime}\left(x_{2} x_{2}^{\prime}\right)\right|\right)+m_{3}\left(m_{1}+\left|N_{\Gamma_{3}}^{\prime}\left(x_{3}\right)\right|-\left|N_{\Gamma_{3}}^{\prime}\left(x_{3} x_{3}^{\prime}\right)\right|\right) \\
& \left(m_{1}+\left|N_{\Gamma_{3}}^{\prime}\left(x_{3}^{\prime}\right)\right|-\left|N_{\Gamma_{3}}^{\prime}\left(x_{3} x_{3}^{\prime}\right)\right|\right)+n_{1} n_{2}\left(m_{3}+n_{2}-1+m_{2}-\left|N_{\Gamma_{2}}^{\prime}\left(x_{2}\right)\right|\right)\left(2 m_{1}+n_{1}-1\right) \\
& +2 m_{1} n_{2}\left(\left(n_{3}+1\right)\left(2 m_{1}+n_{1}-1\right)-2\left(m_{3}+n_{2}-1+m_{2}-\left|N_{\Gamma_{2}}^{\prime}\left(x_{2}\right)\right|\right)\right) \\
& +2 m_{2} n_{1}\left(m_{3}+n_{2}+m_{2}-\left|N_{\Gamma_{2}}^{\prime}\left(x_{2}\right)\right|-2 m_{1}-n_{1}\right)-2 n_{2}\left(n_{3}+1\right) M_{1}\left(\Gamma_{1}\right) \\
& +4 m_{1} m_{2}\left(n_{3}+3\right)-n_{1} M_{1}\left(\Gamma_{2}\right)+m_{1} n_{3}\left(\left(2 n_{2}+m_{2}+n_{3}+m_{3}+1-\left|N_{\Gamma_{3}}^{\prime}\left(x_{3}\right)\right|\right)\left(3 m_{1}-1\right)\right. \\
& -n_{3}\left(2 n_{2}+m_{2}+n_{3}+m_{3}+1-\left|N_{\Gamma_{3}}^{\prime}\left(x_{3}\right)\right|\right) M_{1}\left(\Gamma_{1}\right)+2 m_{1} m_{3}\left(2 n_{2}+m_{2}+n_{3}+m_{3}-3 m_{1}\right. \\
& \left.+2-\left|N_{\Gamma_{3}}^{\prime}\left(x_{3}\right)\right|\right)+2 m_{3} M_{1}\left(\Gamma_{1}\right)-m_{1} M_{1}\left(\Gamma_{3}\right)+n_{1} m_{1}\left(m_{2}+n_{1} n_{2}-n_{2}-2\right) \\
& \left(m_{3}+m_{1} n_{3}\right)+n_{1}\left(m_{2}+n_{1} n_{2}-n_{2}-2\right) \sum_{x_{1} x \prime_{1} \in E\left(\Gamma_{1}\right)} d_{\Gamma_{1}}\left(x_{1}^{\prime}\right)+4 m_{1}^{2}\left(m_{3}+m_{1} n_{3}\right)+2 n_{1} M_{2}\left(\Gamma_{1}\right) \text {. }
\end{aligned}
$$

This completes the proof.

\subsection{Weighted vertex Padmakar-Ivan and weighted vertex Szeged indices}

Now we calculate the weighted versions of vertex PI and vertex Szeged indices of SVE join of graph.

\section{Theorem 6.5}

Let $\Gamma_{1}, \Gamma_{2}$, and $\Gamma_{3}$ be three graphs. Then:

$$
\begin{aligned}
w P I_{v}\left(\Gamma_{1}^{\mathrm{S}} \triangleright\left(\Gamma_{2}^{\mathrm{V}} \cup \Gamma_{3}^{\mathrm{I}}\right)\right) & =F\left(\Gamma_{2}\right)+F\left(\Gamma_{3}\right)+2\left(M_{2}\left(\Gamma_{2}\right)+M_{2}\left(\Gamma_{3}\right)\right)-\left(2\left|N_{\Gamma_{2}}\left(x_{2} x^{\prime}\right)\right|-n_{1}\right) M_{1}\left(\Gamma_{2}\right) \\
& -\left(2\left|N_{\Gamma_{3}}\left(x_{3} x^{\prime}{ }_{3}\right)\right|-m_{1}\right) M_{1}\left(\Gamma_{3}\right)-2 n_{1} m_{2}\left(2\left|N_{\Gamma_{2}}\left(x_{2} x_{2}^{\prime}\right)\right|+n_{1}+n_{2}\right) \\
& -2 m_{1} m_{3}\left(2\left|N_{\Gamma_{3}}\left(x_{3} x^{\prime}\right)\right|-n_{1}-n_{1}+2\right)-4 m_{1} m_{2}+\left(n_{1}+n_{2}+n_{3}+m_{1}\right) \\
& \left(m_{1} n_{3}\left(n_{3}+n_{1}+m_{1}+2\right)+n_{1} n_{2}\left(n_{1}+n_{2}+m+1\right)+2 m_{1}\left(n_{1}+m_{1}\right)\right. \\
& \left.+2\left(n_{1} m_{2}+n_{2} m_{1}\right)\right) .
\end{aligned}
$$

Proof.

$$
\begin{aligned}
w P I_{v}\left(\Gamma_{1}^{\mathrm{S} \triangleright}\left(\Gamma_{2}^{\mathrm{V}} \cup \Gamma_{3}^{\mathrm{I}}\right)\right) & =\sum_{u v \in E\left(\Gamma_{2}\right)}\left(d_{\Gamma_{2}}\left(x_{2}\right)+d_{\Gamma_{2}}\left(x_{2}^{\prime}\right)+2 n_{1}\right)\left(d_{\Gamma_{2}}\left(x_{2}\right)+d_{\Gamma_{2}}\left(x_{2}^{\prime}\right)\right. \\
& \left.-2\left|N_{\Gamma_{2}}\left(x_{2} x_{2}^{\prime}\right)\right|\right)+\sum_{u v \in E\left(\Gamma_{3}\right)}\left(d_{\Gamma_{3}}\left(x_{3}\right)+d_{\Gamma_{3}}\left(x_{3}^{\prime}\right)+2 m_{1}\right)\left(d_{\Gamma_{3}}\left(x_{3}\right)\right. \\
& \left.+d_{\Gamma_{3}}\left(x_{3}^{\prime}\right)-2\left|N_{\Gamma_{3}}\left(x_{3} x_{3}^{\prime}\right)\right|\right)+\sum_{x_{1} \in V\left(\Gamma_{1}\right)} \sum_{x_{2} \in V\left(\Gamma_{2}\right)}\left(d_{\Gamma_{1}}\left(x_{1}\right)\right. \\
& \left.+d_{\Gamma_{2}}\left(x_{2}\right)+n_{1}+n_{2}\right)\left(n_{1}+n_{2}+n_{3}+m_{1}-d_{\Gamma_{2}}\left(x_{2}\right)\right) \\
& +\sum_{x_{1} \in E\left(\Gamma_{1}\right)} \sum_{x_{3} \in V\left(\Gamma_{3}\right)}\left(n_{3}+m_{1}+2+d_{\Gamma_{3}}\left(x_{3}\right)\right)\left(n_{1}+n_{2}+n_{3}+m_{1}-d_{\Gamma_{3}}\left(x_{3}\right)\right) \\
& +\sum_{x_{1} \in V\left(\Gamma_{1}\right)} \sum_{x_{1} \in E\left(\Gamma_{1}\right)}\left(d_{\Gamma_{1}}\left(x_{1}\right)+n_{2}+n_{3}+2\right)\left(n_{1}+n_{2}+n_{3}+m_{1}\right)
\end{aligned}
$$




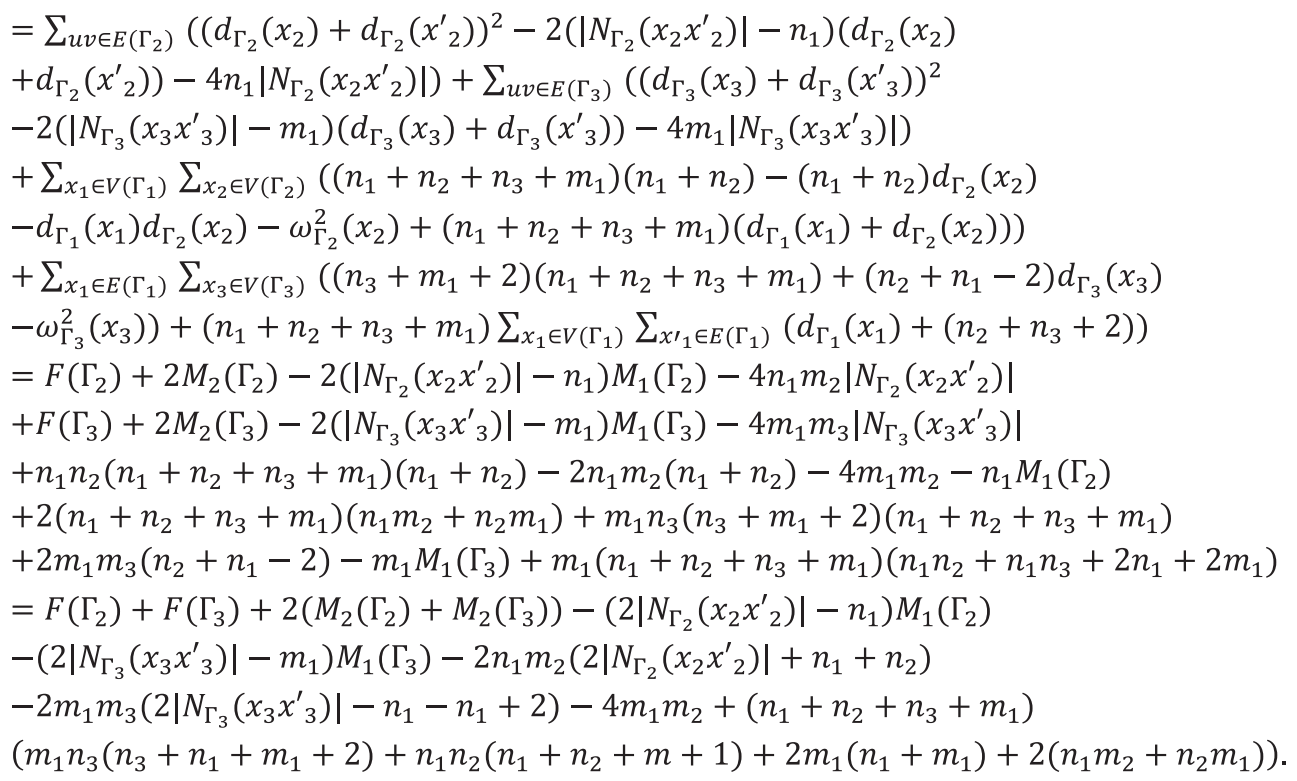

This completes the proof.

\section{Theorem 6.6}

Let $\Gamma_{1}, \Gamma_{2}$, and $\Gamma_{3}$ be three graphs. Then:

$$
\begin{aligned}
w S z_{v}\left(\Gamma_{1}^{\mathrm{S} \triangleright}\left(\Gamma_{2}^{\mathrm{V}} \cup \Gamma_{3}^{\mathrm{I}}\right)\right) & =Z_{2,1}\left(\Gamma_{2}\right)+Z_{2,1}\left(\Gamma_{3}\right)-\left|N_{\Gamma_{2}}\left(x_{2} x_{2}^{\prime}\right)\right| F\left(\Gamma_{2}\right)-\left|N_{\Gamma_{3}}\left(x_{3} x_{3}^{\prime}\right)\right| F\left(\Gamma_{3}\right) \\
& -\left(n_{2}+m_{1}\right) F\left(\Gamma_{1}\right)+2\left(n_{1}-\left|N_{\Gamma_{2}}\left(x_{2} x_{2}^{\prime}\right)\right|\right) M_{2}\left(\Gamma_{2}\right)+2\left(m_{1}-\left|N_{\Gamma_{3}}\left(x_{3} x^{\prime}{ }_{3}\right)\right|\right. \\
& M_{2}\left(\Gamma_{3}\right)\left(n_{2}\left(2 n_{2}+n_{3}+3 m_{1}\right)+m_{1}\left(n_{1}-2-m_{1}\right)\right) M_{1}\left(\Gamma_{1}\right)-\left(\left|N_{\Gamma_{2}}\left(x_{2} x_{2}^{\prime}\right)\right|\right. \\
& \left.\left(\left|N_{\Gamma_{2}}\left(x_{2} x_{2}^{\prime}\right)\right|+2 n_{2}\right)+n_{1}+n_{1} m_{1}+2 m_{1}\right) M_{1}\left(\Gamma_{2}\right)-\left(\left|N_{\Gamma_{2}}\left(x_{2} x_{2}^{\prime}\right)\right|\right. \\
& \left.\left(\left|N_{\Gamma_{2}}\left(x_{2} x_{2}^{\prime}\right)\right|+2 m_{2}\right)+m_{1}\left(n_{1}+m_{1}-2\right)\right) M_{1}\left(\Gamma_{3}\right)+2 m_{1} n_{2}\left(n_{1} n_{2}+m_{1} n_{3}\right. \\
& \left.+n_{2}^{2}-n_{2} n_{3}-n_{1}^{2}-n_{1} m_{1}\right)+2 n_{1} m_{2}\left(\left(n_{1}+m_{1}\right)\left(n_{1}+2 n_{2}+n_{3}\right)-\left|N_{\Gamma_{2}}\left(x_{2} x_{2}^{\prime}\right)\right|\right) \\
& +n_{1} n_{2}\left(n_{1}+n_{2}\right)\left(n_{2}+n_{3}\right)\left(n_{1}+m_{1}\right)+m_{1} n_{3}\left(n_{1}+m_{1}-2\right)\left(n_{1}+m_{1}+2\right) \\
& \left(n_{2}+n_{3}+2\right)-2 m_{1} m_{3}\left(\left(n_{1}+m_{1}-2\right)\left(m_{1}-n_{2}\right)-\left|N_{\Gamma_{3}}\left(x_{3} x_{3}^{\prime}\right)\right|+n_{1} m_{1}\left(n_{3}\right.\right. \\
& \left.+m_{1}+2\right)\left(n_{2}+n_{3}+2\right)\left(n_{1}+n_{2}-2\right)+2 m_{1}^{2}\left(n_{3}+m_{1}+2\right)\left(n_{1}+2 n_{2}+n_{3}\right) .
\end{aligned}
$$

Proof.

$$
\begin{aligned}
& w S z_{v}\left(\Gamma_{1}^{S} \triangleright\left(\Gamma_{2}^{\mathrm{V}} \cup \Gamma_{3}^{\mathrm{I}}\right)\right)=\sum_{u v \in E\left(\Gamma_{2}\right)}\left(d_{\Gamma_{2}}\left(x_{2}\right)+d_{\Gamma_{2}}\left(x_{2}^{\prime}{ }_{2}\right)+2 n_{1}\right)\left(d_{\Gamma_{2}}\left(x_{2}\right)-\left|N_{\Gamma_{2}}\left(x_{2} x_{2}^{\prime}\right)\right|\right) \\
& \left(d_{\Gamma_{2}}\left(x_{2}^{\prime}\right)-\left|N_{\Gamma_{2}}\left(x_{2} x_{2}^{\prime}\right)\right|\right)+\sum_{u v \in E\left(\Gamma_{3}\right)}\left(d_{\Gamma_{3}}\left(x_{3}\right)+d_{\Gamma_{3}}\left(x_{3}^{\prime}\right)+2 m_{1}\right) \\
& \left(d_{\Gamma_{3}}\left(x_{3}\right)-\left|N_{\Gamma_{3}}\left(x_{3} x^{\prime}{ }_{3}\right)\right|\right)\left(d_{\Gamma_{3}}\left(x^{\prime}{ }_{3}\right)-\left|N_{\Gamma_{3}}\left(x_{3} x^{\prime}{ }_{3}\right)\right|\right) \\
& +\sum_{x_{1} \in V\left(\Gamma_{1}\right)} \sum_{x_{2} \in V\left(\Gamma_{2}\right)}\left(d_{\Gamma_{1}}\left(x_{1}\right)+d_{\Gamma_{2}}\left(x_{2}\right)+n_{1}+n_{2}\right)\left(n_{2}+n_{3}+d_{\Gamma_{1}}\left(x_{1}\right)\right. \\
& \left.-d_{\Gamma_{2}}\left(x_{2}\right)\right)\left(n_{1}+m_{1}-d_{\Gamma_{1}}\left(x_{1}\right)\right)+\sum_{x_{1} \in E\left(\Gamma_{1}\right)} \sum_{x_{3} \in V\left(\Gamma_{3}\right)}\left(n_{3}+m_{1}+2\right. \\
& \left.+d_{\Gamma_{3}}\left(x_{3}\right)\right)\left(n_{2}+n_{3}+2-d_{\Gamma_{3}}\left(x_{3}\right)\right)\left(n_{1}+m_{1}-2\right) \\
& +\sum_{x_{1} \in V\left(\Gamma_{1}\right)} \sum_{x^{\prime} \in E\left(\Gamma_{1}\right)}\left(d_{\Gamma_{1}}\left(x_{1}\right)+n_{2}+n_{3}+2\right)\left(n_{2}+n_{1}-2+d_{\Gamma_{1}}\left(x_{1}\right)\right) \\
& \left(n_{3}+m_{1}+2-d_{\Gamma_{1}}\left(x_{1}\right)\right) \\
& =\sum_{u v \in E\left(\Gamma_{2}\right)}\left(\left(\omega_{\Gamma_{2}}^{2}\left(x_{2}\right) d_{\Gamma_{2}}\left(x_{2}^{\prime}\right)+d_{\Gamma_{2}}\left(x_{2}\right) \omega_{\Gamma_{2}}^{2}\left(x_{2}^{\prime}\right)\right)-\left|N_{\Gamma_{2}}\left(x_{2} x_{2}^{\prime}\right)\right|\right. \\
& \left(d_{\Gamma_{2}}\left(x_{2}\right)+d_{\Gamma_{2}}\left(x_{2}^{\prime}\right)\right)^{2}-\left|N_{\Gamma_{2}}\left(x_{2} x_{2}^{\prime}\right)\right|^{2}\left(d_{\Gamma_{2}}\left(x_{2}\right)+d_{\Gamma_{2}}\left(x_{2}^{\prime}\right)\right) \\
& +2 n_{1} d_{\Gamma_{2}}\left(x_{2}\right) d_{\Gamma_{2}}\left(x_{2}^{\prime}\right)-2 n_{1}\left|N_{\Gamma_{2}}\left(x_{2} x_{2}^{\prime}\right)\right|\left(d_{\Gamma_{2}}\left(x_{2}\right)+d_{\Gamma_{2}}\left(x_{2}^{\prime}\right)\right) \\
& -2 n_{1} \mid N_{\Gamma_{2}}\left(\left.x_{2} x_{2}^{\prime}{ }_{2}\right|^{2}\right)+\sum_{u v \in E\left(\Gamma_{3}\right)}\left(\left(\omega_{\Gamma_{3}}^{2}\left(x_{3}\right) d_{\Gamma_{3}}\left(x_{3}^{\prime}\right)+d_{\Gamma_{3}}\left(x_{3}\right) \omega_{\Gamma_{3}}^{2}\left(x_{3}^{\prime}{ }_{3}\right)\right)\right. \\
& -\left|N_{\Gamma_{3}}\left(x_{3} x^{\prime}{ }_{3}\right)\right|\left(d_{\Gamma_{3}}\left(x_{3}\right)+d_{\Gamma_{3}}\left(x^{\prime}{ }_{3}\right)\right)^{2}-\left|N_{\Gamma_{3}}\left(x_{3} x^{\prime}{ }_{3}\right)\right|^{2}\left(d_{\Gamma_{3}}\left(x_{3}\right)\right.
\end{aligned}
$$




$$
\begin{aligned}
& \left.+d_{\Gamma_{3}}\left(x_{3}^{\prime}\right)\right)+2 m_{1} d_{\Gamma_{3}}\left(x_{3}\right) d_{\Gamma_{3}}\left(x^{\prime}{ }_{3}\right)-2 m_{1}\left|N_{\Gamma_{3}}\left(x_{3} x_{3}^{\prime}\right)\right|\left(d_{\Gamma_{3}}\left(x_{3}\right)\right. \\
& \left.\left.+d_{\Gamma_{3}}\left(x_{3}^{\prime}\right)\right)-2 m_{1}\left|N_{\Gamma_{3}}\left(x_{3} x_{3}^{\prime}\right)\right|^{2}\right)+\sum_{x_{1} \in V\left(\Gamma_{1}\right)} \sum_{x_{2} \in V\left(\Gamma_{2}\right)}\left(-\omega_{\Gamma_{1}}^{3}\left(x_{1}\right)\right. \\
& -\left(2 n_{2}+n_{3}-m_{1}\right) \omega_{\Gamma_{1}}^{2}\left(x_{1}\right)-\left(n_{1}+m_{1}\right) \omega_{\Gamma_{2}}^{2}\left(x_{2}\right)+d_{\Gamma_{1}}\left(x_{1}\right) \omega_{\Gamma_{2}}^{2}\left(x_{2}\right) \\
& +\left(\left(n_{2}+n_{3}\right)\left(n_{1}+m_{1}\right)-\left(n_{2}+n_{3}-n_{1}-m_{1}\right)\left(n_{1}+n_{2}\right)\right) d_{\Gamma_{1}}\left(x_{1}\right)+\left(n_{1}+m_{1}\right) \\
& \left(n_{1}+2 n_{2}+n_{3}\right) d_{\Gamma_{2}}\left(x_{2}\right)-\left(n_{3}-n_{1}\right) d_{\Gamma_{1}}\left(x_{1}\right) d_{\Gamma_{2}}\left(x_{2}\right)+\left(n_{1}+n_{2}\right)\left(n_{2}+n_{3}\right) \\
& \left.\left(n_{1}+m_{1}\right)\right)+\left(n_{1}+m_{1}-2\right) \sum_{x_{1} \in E\left(\Gamma_{1}\right)} \sum_{x_{3} \in V\left(\Gamma_{3}\right)}\left(\left(n_{3}+m_{1}+2\right)\left(n_{2}+n_{3}+2\right)\right. \\
& \left.-\left(m_{1}-n_{2}\right) d_{\Gamma_{3}}\left(x_{3}\right)-\omega_{\Gamma_{3}}^{2}\left(x_{3}\right)\right)+\sum_{x_{1} \in V\left(\Gamma_{1}\right)} \sum_{x^{\prime}{ }_{1} \in E\left(\Gamma_{1}\right)}\left(\left(n_{2}+n_{3}+2\right)\right. \\
& \left(n_{2}+n_{1}-2\right)\left(n_{3}+m_{1}+2\right)+\left(\left(n_{1}+2 n_{2}+n_{3}\right)\left(n_{3}+m_{1}+2\right)-\left(n_{2}+n_{1}-2\right)\right. \\
& \left.\left.\left(n_{2}+n_{3}+2\right)\right) d_{\Gamma_{1}}\left(x_{1}\right)+\left(m_{1}-n_{1}-2 n_{2}+2\right) \omega_{\Gamma_{1}}^{2}\left(x_{1}\right)-\omega_{\Gamma_{1}}^{3}\left(x_{1}\right)\right) \\
& =Z_{2,1}\left(\Gamma_{2}\right)+Z_{2,1}\left(\Gamma_{3}\right)-\left|N_{\Gamma_{2}}\left(x_{2} x_{2}^{\prime}\right)\right| F\left(\Gamma_{2}\right)-\left|N_{\Gamma_{3}}\left(x_{3} x_{3}^{\prime}\right)\right| F\left(\Gamma_{3}\right) \\
& -\left(n_{2}+m_{1}\right) F\left(\Gamma_{1}\right)+2\left(n_{1}-\left|N_{\Gamma_{2}}\left(x_{2} x_{2}^{\prime}\right)\right|\right) M_{2}\left(\Gamma_{2}\right)+2\left(m_{1}-\left|N_{\Gamma_{3}}\left(x_{3} x_{3}^{\prime}\right)\right| M_{2}\left(\Gamma_{3}\right)\right. \\
& \left(n_{2}\left(2 n_{2}+n_{3}+3 m_{1}\right)+m_{1}\left(n_{1}-2-m_{1}\right)\right) M_{1}\left(\Gamma_{1}\right) \\
& -\left(\left|N_{\Gamma_{2}}\left(x_{2} x_{2}^{\prime}\right)\right|\left(\left|N_{\Gamma_{2}}\left(x_{2} x_{2}^{\prime}\right)\right|+2 n_{2}\right)+n_{1}+n_{1} m_{1}+2 m_{1}\right) M_{1}\left(\Gamma_{2}\right) \\
& -\left(\left|N_{\Gamma_{2}}\left(x_{2} x_{2}^{\prime}\right)\right|\left(\left|N_{\Gamma_{2}}\left(x_{2} x_{2}^{\prime}\right)\right|+2 m_{2}\right)+m_{1}\left(n_{1}+m_{1}-2\right)\right) M_{1}\left(\Gamma_{3}\right) \\
& +2 m_{1} n_{2}\left(n_{1} n_{2}+m_{1} n_{3}+n_{2}^{2}-n_{2} n_{3}-n_{1}^{2}-n_{1} m_{1}\right) \\
& +2 n_{1} m_{2}\left(\left(n_{1}+m_{1}\right)\left(n_{1}+2 n_{2}+n_{3}\right)-\left|N_{\Gamma_{2}}\left(x_{2} x_{2}^{\prime}\right)\right|\right) \\
& +n_{1} n_{2}\left(n_{1}+n_{2}\right)\left(n_{2}+n_{3}\right)\left(n_{1}+m_{1}\right)+m_{1} n_{3}\left(n_{1}+m_{1}-2\right) \\
& \left(n_{1}+m_{1}+2\right)\left(n_{2}+n_{3}+2\right)-2 m_{1} m_{3}\left(\left(n_{1}+m_{1}-2\right)\left(m_{1}-n_{2}\right)\right. \\
& -\left|N_{\Gamma_{3}}\left(x_{3} x_{3}^{\prime}\right)\right|+n_{1} m_{1}\left(n_{3}+m_{1}+2\right)\left(n_{2}+n_{3}+2\right)\left(n_{1}+n_{2}-2\right) \\
& +2 m_{1}^{2}\left(n_{3}+m_{1}+2\right)\left(n_{1}+2 n_{2}+n_{3}\right) \text {. }
\end{aligned}
$$

This completes the proof.

\section{Conclusion}

The operation of subdivision vertex-edge join of graphs is also known as SVE join of graphs. We computed the several distance based topological descriptors of graphs, namely vertex Padmakar-Ivan $\left(P I_{v}\right)$ index, vertex Szeged $\left(S z_{v}\right)$ index, edge Padmakar-Ivan $(P I)$ index, edge Szeged $(S z)$ index, weighted vertex PadmakarIvan $\left(w P I_{v}\right)$ index, and weighted vertex Szeged $\left(w S z_{v}\right)$ index of SVE join of graphs. These results are helpful for people who are interested in applied chemistry for the computation of SVE join of graphs.

Acknowledgement: The authors are very grateful to the referees for their careful reading with corrections and useful comments, which improved this work very much.

Funding information: Authors state no funding involved

Author contributions: Syed Sheraz Asghar: writing original draft; Muhammad Ahsan Binyamin: writing - review and editing; Shehnaz Akhtar and Mehar Ali Malik: methodology; Yu-Ming Chu: formal analysis, visualization, resources.

Conflict of interest: Authors state no conflict of interest.

\section{References}

Ashrafi A.R., Doslić T., Hamzeh A., The Zagreb coindices of graph operations. Discrete Appl. Math., 2010, 158, 1571-1578.

Azari M., Iranmanesh A., Computing the eccentric-distance sum for graph operations. Discrete Appl. Math., 2013, 161, 2827-2840.

Bajaj S., Sambi S.S., Gupta S., Madan A.K., Model for prediction of anti-HIV activity of 2-pyridinone derivatives using novel topological descriptor. QSAR Comb. Sci., 2006, 25, 813-823.

Balaban A.T., Motoc I., Bonchev D., Mekenyan 0. Topological indices for structure-activity correlations. In Steric effects in drug design. Springer, Berlin, Heidelberg, 1983.

Balasubramanian K., Applications of combinatorics and graph theory to spectrosocpy and quantum chemistry. Chem. Rev., 1985a, 85(6), 599-618.

Balasubramanian K., Characteristic polynomials of organic polymer sand periodic structure. J. Comput. Chem., 1985b, 6(6), 656-661.

Basak S.C., Grunwald G.D., Gute B.D., Balasubramanian K., Opitz D., Use of statistical and neural net approaches in predicting toxicity of chemicals. J. Chem. Inf. Comput. Sci., 2000, 40(4), 885-890.

Das K.C., Yurttas A., Togan M., Cevik A.S., Cangul I.N., The multiplicative Zagreb indices of graph operations. J. Inequal. Appl., 2013, 90, 1-14.

De N., Nayeem S. M.A., Pal A., The F-coindex of some graph operations. SpringerPlus, 2016, 5(1), 221.

Devillers J., Balaban A.T., Topological indices and related descriptors in QSAR and QSPR. Gordon and Breach, Amsterdam, 1999. 
Diudea M.V., QSPR/QSAR Studies by Molecular Descriptors. Huntington, New York, Nova, 2001.

Dobrynin A.A., Entringer R., Gutman I., Wiener index of trees: Theory and applications. Acta Appl. Math., 2001, 66, 211-249.

Gupta S., Singh M., Madan A.K., Application of graph theory: Relationship of eccentric connectivity index and Wiener's index with anti-inflammatory activity. J. Math. Anal. Appl., 2002, 266, 259-268.

Gutman I., A formula for the Wiener number of trees and its extension to graphs containing cycles. Graph Theory Notes NY, 1994, 27, 9-15.

Gutman I., Ashrafi A.R., The edge version of the Szeged index. Croat. Chem. Acta, 81, 2008, 81, 263-266.

Gutman I., Khadikar P.V., Rajput P.V., Karmarkar S., The Szeged index of polyacenes. J. Ser. Chem. Soc., 1995, 60, 759-764.

Hansch C., Leo L., Exploring QSAR fundamentals and applicability in chemistry and biology. Amererican Chemical Society, Washington DC, 1996.

Ilic A., Milosavljevic N., The weighted vertex PI index. Math. Comp. Model., 2013, 57(3), 623-631.

Imran M., Baig A. Q., Ali H., Rehman S. U., On topological properties of poly honeycomb networks. Period. Math. Hung., 2016, 73(1), 100-119.

Khadikar P.V., Karmarkar S., Agrawal V.K., A novel PI index and its applications to QSPR/QSAR studies. J. Chem. Inf. Comput. Sci., 2001, 41(4), 934-949.

Khalifeh M. H., Yousefi-Azaria H., Ashrafi A.R., Vertex and edge PI indices of Cartesian product graphs. Discrete Appl. Math., 2008, 156, 1780-1789.

Khalifeh M.H., Yousefi-Azaria H., Ashrafi A.R., The first and second Zagreb indices of some graph operations. Discrete Appl. Math., 2009, 157, 804-811.

Klavžar S., Rajapakse A., Gutman I., The Szeged and the Wiener index of graphs. Appl. Math. Lett., 1996, 9(5), 45-49.
Liu J.B., Ali B., Malik M.A., Siddiqui H.M.A., Imran M., Reformulated Zagreb indices of some derived graphs. Mathematics, 2019, 7(4), 366.

Nagarajan S., Pattabiraman K., Chandrasekharan M., Weighted Szeged Index of Generalized Hierarchical Product of Graphs. Gen. Math. Notes, 2014, 23(2), 85-95.

Pattabiraman K., Kandan P., Weighted Szeged indices of some graph operations. Transactions on Combinatorics, 2016, 5(1), 25-35.

Pattabiraman K., Paulraja P., Vertex and edge Padmakar-Ivan indices of the generalized hierarchical product of graphs. Discrete Appl. Math., 2012, 160, 1376-1384.

Platt J.R., Prediction of isomeric differences in paraffin properties. J. Chem. Phys., 1952, 56, 328-336.

Randić M., Wiener-Hosoya index: A novel graph theoretical molecular descriptor. J. Chem. Inf. Comput. Sci., 2004, 44, 373-377.

Siddiqui H.M.A., Computation of Zagreb indices and Zagreb polynomials of Sierpinski graphs. Hacet. J. Math. Stat., 2020, 49(2), 754-765.

Tavakoli M., Rahbarnia F., The vertex and edge pi indices of generalized hierarchical product of graphs. J. Appl. Math. Inf., 2013, 31(3-4), 469-477.

Wen F., Zhang Y., Li M., Spectra of subdivision vertex-edge join of three graphs. Mathematics, 2019, 7, 171.

Wiener M., Structural determination of paraffin boiling points. J. Am. Chem. Soc., 1947, 69, 17-20.

Xu J., Liu J.B., Bilal A., Ahmad U., Siddiqui H.M.A., Ali B., et al., Distance degree index of some derived graphs. Mathematics, 2019, 7(3), 283.

Yang H., Imran M., Akhter S., Iqbal Z., Siddiqui M.K., On distancebased topological descriptors of subdivision vertex-edge join of three graphs. IEEE Access, 2019, 7, 143381-143391.

Yousefi-Azari H., Manoochehrian V., Ashrafi A.R., The PI index of product graphs. Appl. Math. Lett., 2008, 21, 624-627. 